\title{
Modelos lineares generalizados mistos na avaliação genética da prenhez precoce na raça Nelore
}

\author{
[Generalized linear mixed models on genetic evaluation of early pregnancy in \\ Nellore breed] \\ D.A. Garcia ${ }^{1}$, I.G. Pereira ${ }^{2}$, F. Fonseca e Silva ${ }^{3}$, A.V. Pires ${ }^{2}$, M.C. Pessoa ${ }^{1}$, R.A.M. Oliveira ${ }^{4}$ \\ ${ }^{1}$ Aluno de pós-graduação - UNESP - Jaboticabal, SP \\ ${ }^{2}$ FCAV-UFVJM - Diamantina, MG \\ ${ }^{3}$ Universidade Federal de Viçosa - Viçosa, MG \\ ${ }^{4}$ Aluno de graduação - UFVJM - Diamantina, MG
}

\begin{abstract}
RESUMO
Utilizaram-se os modelos lineares generalizados com as funções de ligação probit e logit na avaliação da prenhez precoce, e observaram-se os efeitos na variabilidade genética e na seleção de reprodutores quando diferentes idades são adotadas na definição dessa característica. A prenhez precoce foi estudada aos 15 (PP15) e 21 meses (PP21). Correlações entre os valores genéticos preditos e a porcentagem de touros em comum, considerando $10 \%$ dos touros com maiores valores genéticos (TOP10), entre a classificação dos modelos com função de ligação logit e probit e em cada modelo entre PP15 e PP21, foram calculadas. As herdabilidades para PP15 e PP21 foram próximas entre os modelos, exceto para PP15 utilizando a função de ligação probit. Aquelas entre os modelos e a TOP/10 foram altas. Os critérios de Akaike e Bayesiano apresentaram-se semelhantes entre os modelos. As correlações entre PP15 e PP21 e a TOP10, considerando o mesmo modelo, foram de média magnitude.
\end{abstract}

Palavras-chave: gado de corte, GLMM, herdabilidade, função de ligação, precocidade

\begin{abstract}
This study aimed to use the generalized linear models with probit and logit link function to evaluate early pregnancy, and to observe the effects on genetic variability and on sire selection when different ages are adopted in the definition of this trait. Early pregnancy was studied at 15 (EP15), and 21 (EP21) months. The analysis was done in $R$ software. Pearson correlations $(P C)$, between genetic predicted values and percentage of bulls in common considering only 10\% of bulls with higher genetic values (TOP 10), between classification by logit and probit models and in each model among EP15 and EP21, were calculated. The heritability for EP15 and EP21 were close between models, except for EP15 using probit link function. PC and TOP10 among models were high. The Akaike and Bayesian criteria reported was similar between models. TOP10, considering the same model, among EP15-EP21 were moderated between EP15-EP21.
\end{abstract}

Keywords: beef cattle heritability, early pregnancy, link function

\section{INTRODUÇÃO}

Em uma empresa agropecuária, em que a bovinocultura de corte é a principal atividade, vários fatores podem determinar a viabilidade econômica do empreendimento. Dentre estes, pode-se citar a capacidade administrativa do empreendedor, mão de obra qualificada, manejo alimentar e sanitário bem estruturado e potencial genético do rebanho. Este deve ser trabalhado visando à seleção de animais para um sistema de produção de ciclo curto. Logo, procura-se imprimir no rebanho precocidade sexual, de crescimento e terminação (Carneiro, 2008).

Recebido em 16 de abril de 2010

Aceito em 2 de agosto de 2011

E-mail: diogo.agarcia@gmail.com

Apoio financeiro: FAPEMIG 
A avaliação genética da precocidade sexual de fêmeas da raça Nelore vem sendo realizada utilizando-se a prenhez precoce como critério de seleção. Essa característica pode ser mensurada facilmente, pois compreende apenas duas categorias, prenhe e não prenhe, não traz custos adicionais ao sistema produtivo, uma vez que o diagnóstico de gestação é uma prática rotineira em gado de corte, e possui variabilidade genética que a potencializa como critério de seleção.

Entretanto, como as características categóricas não atendem às pressuposições dos modelos clássicos de avaliação genética, outros métodos estatísticos mais adequados podem ser utilizados visando a um potencial aumento na habilidade de estimação dos parâmetros genéticos e à predição dos valores genéticos dos animais.

Dentre estes métodos, destacam-se os modelos lineares generalizados mistos (GLMM) (Thompson, 1979; Gianola e Foulley, 1983; Harville e Mee, 1984; Gilmour et al., 1985, 1987; Templeman, 1998; Kadarmideen et al., 2004; Pereria et al., 2006, 2007), os quais podem ser utilizados para a análise de características que seguem distribuições de probabilidade pertencentes à família exponencial. Os GLMM permitem, por meio da utilização de funções de ligação modelar, a probabilidade de estas características assumirem determinados valores.

A função de ligação mais difundida na área de melhoramento animal é a probit, conhecida como modelo threshold (Gianola e Foulley, 1983; Abdel-Azim e Berger, 1999; Kadarmideen et al., 2000, 2001; Silva et al., 2005; Shiotsuki et al., 2009), porém existem outras funções que podem ser exploradas, como a logit, que é amplamente utilizada na área de biometria (Breslow e Clayton, 1993; Demétrio, 2001; Nunes et al., 2004).

Além desse tratamento metodológico especial que esta característica requer, ela vem sendo definida de forma distinta entre os trabalhos realizados na raça Nelore. Eler et al. (2002, 2004) e Silva et al. (2003) definiram como fêmea precoce os animais que apresentaram diagnóstico de gestação positivo ao iniciarem a estação de monta com idade média de 14 meses. Silva et al. (2005) e Shiotsuki et al. (2009) caracterizaram como precoces as novilhas que pariram com idade inferior a 31 meses, ou seja, animais que se tornaram prenhes com até 21 meses. Essas diferentes idades adotadas na definição da expressão fenotípica deste critério de seleção são importantes para melhor atender à bovinocultura de corte brasileira, pois a grande maioria das empresas agropecuárias não apresenta potencial técnico e tecnológico para a exposição das novilhas à reprodução muito jovem, como aos 14 meses de idade. Logo, a avaliação da variabilidade genética da prenhez precoce em idades diferentes e a observação do reflexo dessas distintas definições na seleção dos animais são de grande importância.

Objetivou-se com este estudo utilizar o modelo linear generalizado misto na estimação de parâmetros genéticos e na predição de valores genéticos dos animais para a característica prenhez precoce, comparando a utilização das funções de ligação probit e logit, além de avaliar os efeitos na variabilidade genética e na seleção de reprodutores quando diferentes idades são adotadas na definição desse critério de seleção.

\section{MATERIAL E MÉTODOS}

O conjunto de dados utilizado no estudo foi de fêmeas da raça Nelore nascidas entre 1993 e 2003, pertencentes à Colonial Agropecuária Ltda. Esta empresa pratica seleção desde a década de 70 e, a partir do ano de 1993, é integrante do Programa de Melhoramento Genético da Raça Nelore. Atualmente, o principal objetivo desta consiste na seleção de animais que apresentam precocidade de crescimento, sexual e no acabamento de carcaça. Uma das características reprodutivas alvo de seleção da empresa é a prenhez precoce.

Neste estudo, a prenhez precoce foi avaliada aos 15 e 21 meses, representada, respectivamente, por PP15 e PP21. Dessa forma, as novilhas que pariram em idades iguais ou abaixo de 24 e 30 meses receberam o escore 1 para prenhez precoce. Nas Tab. 1 e 2, descreve-se o banco de dados das distintas idades utilizadas na avaliação da prenhez precoce.

Os GLMM podem ser definidos a partir da especificação de três componentes: i) um componente aleatório, representado pelas variáveis aleatórias independentes pertencentes a uma mesma distribuição da família exponencial, tais como normal, binomial e Poisson; ii) um 
componente sistemático denominado preditor linear, no qual as variáveis explicativas são representadas como uma soma linear de seus efeitos; iii) uma função de ligação que relaciona os componentes aleatório e sistemático, ou seja, associando a média ao preditor linear. Portanto, os efeitos fixos e aleatórios, que influenciam a característica de interesse, são modelados a partir do preditor linear, descrito por:

$$
\boldsymbol{\eta}=X \boldsymbol{\beta}+Z \boldsymbol{u}, \text { em que: }
$$

$\boldsymbol{\eta}$ é o preditor linear; $\boldsymbol{\beta}$ é o vetor de efeitos dos grupos de contemporâneos que foram compostos por fazenda, ano e estação de nascimento (águas e secas); $\boldsymbol{u}$ é o vetor dos valores genéticos aditivos; $X$ e $Z$ são, respectivamente, as matrizes de incidência dos efeitos $\boldsymbol{\beta}$ e $\boldsymbol{u}$.

Tabela 1. Número de touros, fêmeas, grupos de contemporâneos (GC), fêmeas por GC e porcentagem de fêmeas precoces (\%FP) em prenhezes aos 15 e 21 meses

\begin{tabular}{ccccccc}
\multirow{2}{*}{ Característica } & \multirow{2}{*}{$\mathrm{N}^{\circ}$ de touros } & \multirow{2}{*}{$\mathrm{N}^{\circ}$ de Fêmeas } & \multirow{2}{*}{$\% \mathrm{FP}$} & \multirow{2}{*}{$\mathrm{N}^{\circ} \mathrm{GC}$} & \multicolumn{2}{c}{$\mathrm{N}^{\circ}$ Fêmeas/GC } \\
\cline { 5 - 7 } & 193 & 1829 & 4,10 & 17 & 18 & 220 \\
PP15 & 239 & 2666 & 13,05 & 35 & 5 & 220 \\
\hline
\end{tabular}

PP15 e PP21: probabilidade de prenhez aos 15 e 21 meses, respectivamente;

Tabela 2. Número de fêmeas avaliadas por ano para prenhezes aos 15 e 21 meses

\begin{tabular}{crc}
\hline Ano & PP15 & PP21 \\
\hline 1993 & 64 & 163 \\
1994 & 0 & 147 \\
1995 & 0 & 184 \\
1996 & 154 & 162 \\
1997 & 112 & 209 \\
1998 & 317 & 324 \\
1999 & 282 & 282 \\
2000 & 184 & 284 \\
2001 & 280 & 280 \\
2002 & 252 & 441 \\
2003 & 184 & 190 \\
\hline
\end{tabular}

${ }^{\mathrm{P}}$ PP15 e PP21: probabilidade de prenhez aos 15 e 21 meses, respectivamente.

Nos GLMM, de acordo com a distribuição de probabilidade da característica analisada, são definidas as funções de ligação apropriadas. Quando a pressuposição de normalidade pode ser assumida, a função de ligação identidade é adotada, com isso, utiliza-se o modelo clássico para a estimação de parâmetros genéticos e predição dos valores genéticos dos animais. Portanto, é importante ressaltar que este modelo é um caso particular dos modelos lineares generalizados mistos. Neste caso, como a prenhez precoce segue distribuição binomial, as funções logit e probit (modelo threshold) foram utilizadas.

Considerando que $\pi_{i}=P\left[Y_{i}=1\right]$ é a probabilidade de a fêmea $i$ ser precoce, as funções de ligação logit e probit, $g($.$) , são$ dadas, respectivamente, pelas expressões [2] e [3]:

$g\left(\pi_{i}\right)=\ln \left(\frac{\pi_{i}}{1-\pi_{i}}\right)$

$g\left(\pi_{i}\right)=\Phi^{-1}\left(\pi_{i}\right)$, em que:

$\Phi^{-1}($ ) é a função inversa da normal padrão acumulada.

Ao se igualar o preditor linear em [1] às funções de ligação [2] e [3], é possível obter as probabilidades de prenhez precoce $\left(\pi_{i}\right)$. Considerando, respectivamente, as funções de ligação logit e probit, tais probabilidades são dadas pelas expressões [4] e [5]: 


\section{Garcia et al.}

$$
\text { (i) } \begin{aligned}
g\left(\pi_{i}\right)=\ln \left(\frac{\pi_{i}}{1-\pi_{i}}\right)=\eta_{i}=x_{i}^{\prime} \boldsymbol{\beta}+u_{i} \\
\ln \left(\frac{\pi_{i}}{1-\pi_{i}}\right)=x_{i}^{\prime} \boldsymbol{\beta}+u_{i} \\
\frac{\pi_{i}}{1-\pi_{i}}=\exp \left(x_{i}^{\prime} \boldsymbol{\beta}+u_{i}\right) \Rightarrow \pi_{i}=\exp \left(x_{i}^{\prime} \boldsymbol{\beta}+u_{i}\right)-\pi_{i} \exp \left(x_{i}^{\prime} \boldsymbol{\beta}+u_{i}\right) \\
\pi_{i}+\pi_{i} \exp \left(x_{i}^{\prime} \boldsymbol{\beta}+u_{i}\right)=\exp \left(x_{i}^{\prime} \boldsymbol{\beta}+u_{i}\right) \Rightarrow \pi_{i}\left[1+\exp \left(x_{i}^{\prime} \boldsymbol{\beta}+u_{i}\right)\right]=\exp \left(x_{i}^{\prime} \boldsymbol{\beta}+u_{i}\right) \\
\pi_{i}=\frac{\exp \left(x_{i}^{\prime} \boldsymbol{\beta}+u_{i}\right)}{1+\exp \left(x_{i}^{\prime} \boldsymbol{\beta}+u_{i}\right)}
\end{aligned}
$$

em que $\Phi($.$) é a distribuição normal padrão. Dessa forma, pela utilização da teoria dos GLMM, é$ possível modelar a probabilidade de sucesso da variável estudada, ou seja, a probabilidade de uma fêmea ser precoce.

Os conjuntos de dados da PP15 e PP21 foram analisados considerando a metodologia dos GLMM por meio do pacote pedigreemm (Vazquez et al., 2010) do software R (R: a linguage..., 2009). Este pacote utiliza no processo de estimação dos parâmetros o método da máxima verossimilhança restrita (REML). No presente estudo, devido à adoção do modelo de touro, as estimativas da variância genética aditiva correspondem a $1 / 4$ do valor estimado para o componente do efeito aleatório.

A fonte variação não explicada pelo modelo na predição da expressão fenotípica, normalmente denominada de resíduo, está inclusa na aleatoriedade dos valores fenotípicos gerados para a fêmea $i$ pela distribuição binomial com probabilidade $\pi_{i}$. Por isso, quando se utiliza a função de ligação probit, a variância residual $\left(\sigma_{e}^{2}\right)$ é desconhecida e, geralmente, é assumida com sendo 1. Este valor é utilizado com o objetivo de tornar a função de verossimilhança identificável (Sorensen e Gianola, 2002). Para a função de ligação logit, a $\sigma_{e}^{2}$ utilizada é uma aproximação da variância da distribuição logística (Southey et al., 2003). Logo, as estimativas de herdabilidade foram obtidas utilizando-se as expressões [6] e [7], que correspondem, respectivamente, às funções de ligação logit e probit:

$$
\begin{aligned}
& \hat{h}^{2}=\frac{4 \hat{\sigma}_{t}^{2}}{\hat{\sigma}_{t}^{2}+\sigma_{e}^{2}}, \text { sendo } \sigma_{e}^{2}=3,1416^{2} / 3 \\
& \hat{h}^{2}=\frac{4 \hat{\sigma}_{t}^{2}}{\hat{\sigma}_{t}^{2}+\sigma_{e}^{2}}, \text { sendo } \sigma_{e}^{2}=1
\end{aligned}
$$

em que $\hat{\sigma}_{t}^{2}$ é a estimativa da variância de touro.

As predições dos valores genéticos aditivos com base em modelos que utilizaram as funções de ligação logit e probit foram comparadas por meio das correlações de Pearson. Além disso, também foi mensurada a porcentagem de coincidência entre a classificação dos modelos utilizados, considerando-se apenas os 10\% dos touros com maiores valores genéticos (TOP10).

A comparação entre os modelos com função de ligação logit e probit foi realizada por meio dos critérios de informação de Akaike (AIC) e Bayesiano de Schwarz (BIC), que permitem a comparação de modelos não aninhados e penalizam modelos mais parametrizados, sendo o $\mathrm{BIC}$ o mais rigoroso, favorecendo modelos mais parcimoniosos (Wolfinger, 1993; Nunez-Antón e Zimmerman, 2000). Os critérios AIC e BIC são 
dados pelas expressões [8] e [9], respectivamente:

$$
\begin{aligned}
& A I C=-2 \log L+2 p \\
& B I C=-2 \log L+p \log (N), \text { em que }
\end{aligned}
$$

$\log L$ é o logaritmo da função de verossimilhança no ponto máximo; $p$ é o número de parâmetros do modelo;

$N$ é o número de observação para prenhez precoce.

\section{RESULTADOS E DISCUSSÃO}

O número de fêmeas e a porcentagem delas que são precoces para PP15 e PP21 foram, respectivamente, $1.829(4,1 \%)$ e $2.666(13,0 \%)$. Eler et al. (2002; 2004), ao trabalharem com prenhez precoce, com definição de acordo com a PP15, observaram 19,8 e $17 \%$ de fêmeas precoces, valores estes bem superiores ao observado neste estudo. Já Silva et al. (2005) e Shiostsuki et al. (2009), ao usarem uma determinação de sucesso para precocidade semelhante à da PP21, obtiveram, respectivamente, 13,3 e $10,4 \%$, porcentagens próximas à observada neste trabalho.

As estimativas de herdabilidade (Tab. 3) para prenhez aos 15 e 21 meses foram de médias a altas. Para PP15, o valor obtido da $\hat{h}^{2}$ na escala logística, 0,64, apresenta-se próximo aos estimados por Eler et al. (2002; 2004), sendo, respectivamente, 0,57 e 0,68 . Silva et al. (2005) e Shiotsuki et al. (2009) observaram valores de $\hat{h}^{2}$ mais baixos que os do presente estudo para PP21, 0,52 e 0,50, enquanto Silva et al. (2003) obtiveram o valor de 0,73. Portanto, os resultados deste estudo corroboram com os trabalhos realizados na raça Nelore para a característica prenhez precoce, que, independentemente da idade de avaliação, apresenta variabilidade genética para ser incluída como característica alvo de seleção em programas de melhoramento, com potencial para se obter ganhos genéticos satisfatórios.

Tabela 3. Estimativas de herdabilidade $\left(\hat{h}^{2}\right)$ obtidas utilizando os modelos com funções de ligação logit e probit

\begin{tabular}{ccc}
\hline \multirow{2}{*}{ Característica } & \multicolumn{3}{c}{ Função de ligação } \\
\cline { 2 - 3 } & Logit & Probit \\
\hline PP15 & 0,64 & 0,47 \\
PP21 & 0,68 & 0,64 \\
\hline
\end{tabular}

${ }^{1}$ PP15 e PP21: probabilidade de prenhez aos 15 e 21 meses, respectivamente.

As diferenças entre as funções de ligação logit e probit nas estimativas de herdabilidade foram pequenas para PP17, entretanto, para prenhez aos 15 meses, os valores foram de 0,64 e 0,47, respectivamente. Garcia (2010), em um estudo de simulação, observou que a maior subestimação da $\hat{h}^{2}$, em relação ao valor paramétrico, obtida com a utilização da função de ligação probit, foi com a porcentagem de fêmeas precoces (\%FP) igual a 5\%, sendo esta a menor porcentagem simulada. Essa subestimação não foi observada quando se utilizou a função de ligação logit. Logo, tal fato pode explicar a diferença observada nas estimativas de $\hat{h}^{2}$ entre as funções de ligação para PP15, pois a \%FP observada foi de $4,1 \%$.

Os valores de correlação de Pearson (Tab. 4) obtidos mostram que os valores genéticos preditos pelos modelos que utilizaram as funções logit e probit são muito próximos para as idades de prenhez precoce estudadas. Seguindo a mesma tendência, a porcentagem de coincidência entre a classificação dos modelos, considerandose os $10 \%$ dos touros com maiores valores genéticos (TOP10), também apresentou resultados expressivos.

Garcia (2010) obteve correlações de Pearson, entre os valores genéticos reais e preditos, e porcentagem de touros que se repetiram no TOP10, entre ranking real e predito, pelos modelos com funções de ligação logit e probit muito próximos uns dos outros, em todos os cenários de simulação estudados. Estes resultados auxiliam na compreensão dos valores expressivos de correlações e da porcentagem de touros em comum no TOP10 obtidos neste estudo para PP15 e PP21. 


\section{Garcia et al.}

Tabela 4. Correlações de Pearson dos valores genéticos preditos e porcentagem de coincidência, considerando os $10 \%$ dos touros com maiores valores genéticos (TOP10), entre os modelos com função de ligação logit e probit

\begin{tabular}{ccc}
\hline Característica & Pearson & TOP10 \\
\hline PP15 & 0,9971 & 89,47 \\
PP21 & 0,9987 & 93,22
\end{tabular}

PP15 e PP21: probabilidade de prenhez aos 15 e 21 meses, respectivamente.

Os valores dos critérios de informação de Akaike e Bayesiano de Schawarz (Tab. 5) para PP15 e PP21 foram muito próximos entre as funções de ligação utilizadas, indicando que o ajuste dos modelos foi semelhante. Esta similaridade de ajuste pode explicar a proximidade dos valores obtidos nas estimativas e predições entre logit e probit.
As correlações de Pearson e as porcentagens de touros coincidentes no TOP10 (Tab. 6), quando se utilizou um modelo com a mesma função de ligação, entre PP15 e PP21, podem ser caracterizadas como de média magnitude, indicando uma diferença considerável entre as predições dos valores genéticos e a classificação dos touros quando suas progênies são desafiadas à reprodução aos 15 e 21 meses de idade.

Tabela 5. Valores dos critérios de informação de Akaike (AIC) e Bayesiano de Schawarz (BIC) para os modelos com função de ligação logit e probit, entre a probabilidade de prenhez aos 15 e 21 meses

\begin{tabular}{cccccc}
\multirow{2}{*}{ Característica } & \multicolumn{3}{c}{ Logit } & & \multicolumn{2}{c}{ Probit } \\
\cline { 2 - 3 } \cline { 5 - 6 } & AIC & BIC & & AIC & BIC \\
\hline PP15 & 566,4 & 663,5 & & 568,0 & 665,0 \\
PP21 & $1.614,0$ & $1.821,0$ & & $1.616,0$ & $1.823,0$
\end{tabular}

PP15 e PP21: probabilidade de prenhez aos 15 e 21 meses, respectivamente.

Considerando os resultados da Tab. 6, é importante que um estudo com uma população mais abrangente de animais da raça Nelore seja realizado para verificar se essa tendência de classificação distinta dos touros será observada. Se esta for confirmada, poderá ser interessante a definição de duas idades para precocidade nas avaliações genéticas, pois poderia atender melhor aos produtores segundo o estágio de desenvolvimento tecnológico da sua criação. Por exemplo, no caso de uma propriedade que se encontra em estágio tecnológico mais avançado, o objetivo poderá ser desafiar as novilhas a reprodução por volta dos 15 meses de idade, porém, em um sistema produtivo menos tecnificado, a meta pode ser aos 21 meses. Portanto, a escolha dos reprodutores utilizados por essas propriedades pode diferir e ser mais adequada aos objetivos do sistema produtivo em questão.

Tabela 6. Correlação de Pearson e porcentagem de coincidência, considerando os $10 \%$ dos touros com maiores valores genéticos (TOP10), nas predições dos modelos com as funções de ligação logit e probit, respectivamente, abaixo e acima da diagonal, entre a probabilidade de prenhez aos 15 e 21 meses

\begin{tabular}{ccccc}
\hline \multirow{2}{*}{ Característica } & \multicolumn{2}{c}{ Correlação } & \multicolumn{2}{c}{ TOP10 } \\
\cline { 2 - 5 } & PP15 & PP21 & PP15 & PP21 \\
\hline PP15 & - & 0,4995 & - & 45,00 \\
PP21 & 0,4926 & - & 50,00 & - \\
\hline
\end{tabular}

PP15,e PP21: probabilidade de prenhez aos 15 e 21 meses, respectivamente. 


\section{CONCLUSÕES}

A prenhez precoce, independentemente da idade de avaliação, apresenta variabilidade genética para ser incluída como característica alvo de seleção em programas de melhoramento, com potencial para se obter ganhos genéticos satisfatórios. Os modelos lineares generalizados mistos que utilizam as funções de ligação probit e logit apresentam estimativas de parâmetros genéticos, predições dos efeitos aleatórios dos animais e ajuste dos modelos muito semelhantes. A correlação entre os valores genéticos e a classificação dos touros foi consideravelmente diferente entre PP15 e PP21. Portanto, seria importante realizar um estudo com uma população mais abrangente de animais da raça Nelore para verificar se prevalece essa tendência na mudança no ranking dos animais.

\section{REFERÊNCIAS}

ABDEL-AZIM, G.A.; BERGER, P.J. Properties of threshold model predictions. J. Anim. Sci., v.77, p.582590, 1999.

BRESLOW, N.E.; CLAYTON, D.G. Approximate inference in generalized linear mixed models. $J$. Am. Stat. Assoc., v.88, p.9-25, 1993.

CARNEIRO, R.L.R. Identificação de animais geneticamente superiores: programa PAINT. In: SIMPÓSIO INTERNACIONAL DE PRODUÇÃO DE GADO DE CORTE, 2., 2008, Viçosa. Anais... Viçosa: UFV, 2008. p.345-358.

DEMETRIO, C.G.B. Modelos lineares generalizados em experimentação agronômica. Piracicaba: ESALQ, 2001. 113p.

ELER, J.P.; SILVA, J.A. II V.; FERRAZ, J.B.S. et al. Genetic evaluation of the probability of pregnancy at 14 months for Nellore heifers. J. Anim. Sci., v.80, p.4951-4954, 2002.

ELER, J.P.; SILVA, J.A.II V.; EVANS, J.L. et al. Additive genetic relationships between heifer pregnancy and scrotal circumference in Nellore cattle. J. Anim. Sci., v.82, p.2519-2527, 2004.

GARCIA, D.A. Avaliação genética da prenhez precoce em animais da raça Nelore utilizando modelos lineares generalizados mistos. 2010. 49f. Trabalho de Conclusão de Curso (Pós-Graduação Strictu Sensu em Zootecnia) - Faculdade de Ciências Agrárias, Departamento de Zootecnia, Universidade Federal dos Vales do Jequitinhonha e Mucuri, Diamantina, MG.
GIANOLA, D.; FOULLEY, J.L. Sire evaluation for ordered categorical data with threshold model. Genet. Select. Evol., v.15, p.201-224, 1983.

GILMOUR, A.R.; ANDERSON, R.D.; RAE, A.L. The analysis of binomial data by generalized linear mixed model. Biometrika, v.72, p.539-599, 1985.

GILMOUR, A.R.; ANDERSON, R.D.; RAE, A.L. Variance components on an underlying scale for ordered multiple threshold categorical data using a generalized linear mixed model. J. Anim. Breed. Genet., v.104, p.149-155, 1987.

HARVILLE, D.A.; MEE, R.W. A mixed model procedure for analysing ordered categorical data. Biometrics, v.40, p.393-408, 1984.

KADARMIDEEN, H.N., THOMPSON, R.; SIMM, G. Linear and threshold model genetic parameters for disease, fertility and milk production in dairy cattle. Anim. Sci., v.71, p.411-419, 2000.

KADARMIDEEN, H.N., REKAYA, R.; GIANOLA, D. Genetic parameters for clinical mastitis in HolsteinFriesians: A Bayesian analysis. Anim. Sci., v.73, p.229-240, 2001.

KADARMIDEEN, H.N., SCHWORER, D.; ILAHI, H. et al. A. Genetics of osteochondral disease and its relationship with meat quality and quantity, growth, and feed conversion traits in pigs. J. Anim. Sci., v.82, p.3118-3127, 2004

NUNES, J.A.R.; MORAIS, A.R.; BUENO FILHO, J.S.S. Modelagem da superdispersão em dados por um modelo linear generalizado misto. Rev. Mat. Estat., v.22, p.55-70, 2004.

NUNEZ-ANTÓN, V.N.; ZIMMERMAN, D.L. Modelling nonstationary longitudinal data. Biometrics, v.56, p.699-705, 2000.

PEREIRA, I.G.; SILVA, F.F.; CARNEIRO, A.P.S. et $a l$. Genetic evaluation of the binary reproductive trait via generalized linear mixed models (GLMM). In: WORLD CONGRESS ON GENETICS APPLIED TO LIVESTOCK PRODUCTION, 2006, Belo Horizonte. Procedings... Belo Horizonte [s.n.]. 2006. v.8. p. 233233.

PEREIRA, I.G.; SILVA, F.F.; GARCIA, D.A. et al. Estimativa de herdabilidade para prenhez de novilhas aos 14 meses em um rebanho Nelore por meio de modelos lineares generalizados mistos. In: REUNIÃO ANUAL DA SOCIEDADE BRASILEIRA DE ZOOTECNIA, 44., 2007, Jaboticabal. Anais... Brasília: SBZ, 2007.

$R$ : a language and environment for statistical computing. Vienna, Austria: 2009. Disponível em:<URL http://www.R-project.org.>. Acessado em: 05 mai. 2009. 


\section{Garcia et al.}

SHIOSTSUKI, L.; SILVA, J.A.II V.; ALBUQUERQUE, L.G. Associação genética da prenhez aos 16 meses com o peso à desmama e o ganho de peso em animais da raça Nelore. Rev. Bras. Zootec., v.38, p.1211-1217, 2009.

SILVA, J.A.II V.; MELIS, M.H.V.; ELER, J.P. et al. Estimação de parâmetros genéticos para probabilidade de prenhez aos 14 meses e altura da garupa em bovinos da raça Nelore. Rev. Bras. Zootec., v.32, p.1141-1146, 2003.

SILVA, J.A.II V.; DIAS L.T.; ALBUQUERQUE L.G. Estudo genético da precocidade sexual de novilhas em um rebanho Nelore. Rev. Bras. Zootec., v.34, p.15681572, 2005.

SOUTHEY, B.R.; RODRIGUEZ-ZAS, S.L.; LEYMASTER, K.A. Discrete time survival analysis of lamb mortality in a terminal sire composite population. J. Anim. Sci., v.81, p.1399-1405, 2003.
SORENSEN, D.; GIANOLA, D. Likelihood, bayesian, and MCMC methods in quantitative genetics. New York: Springer-Verlag, 2002. p.607.

TEMPLEMAN, R.J. Generalized linear mixed models in dairy cattle breeding. J. Dairy Sci., v.81, p.14281444, 1998.

THOMPSON, R. Sire evaluation. Biometrics, v.35, p.339-353, 1979.

VAZQUEZ, A.I.; BATES, D.M.; ROSA, G.J.M. et al. Technical note: an $\mathrm{R}$ package for fitting generalized linear mixed models in animal breeding. J. Anim. Sci., v.88, p.497-504, 2010.

WOLFINGER, R. Covariance structure selection in general mixed models. Comm. Stat. Simul., v.22, p.1079-1106, 1993. 